\title{
Cominimaxness of certain general local cohomology modules
}

\author{
Moharram Aghapournahr (D) \\ Department of Mathematics, Faculty of Science, Arak University, Arak, 38156-8-8349, Iran
}

\begin{abstract}
Let $R$ be a commutative Noetherian ring, $\Phi$ a system of ideals of $R$ and $I \in \Phi$. Let $t \in \mathbb{N}_{0}$ be an integer and $M$ an $R$-module such that $\operatorname{Ext}_{R}^{i}(R / I, M)$ is minimax for all $i \leq t+1$. We prove that if the $R$-module $\mathrm{H}_{\Phi}^{i}(M)$ is $\mathrm{FD}_{\leq 1}$ (or weakly Laskerian) for all $i<t$, then $\mathrm{H}_{\Phi}^{i}(M)$ is $\Phi$-cominimax for all $i<t$ and for any $\mathrm{FD}_{\leq 0}$ (or minimax) submodule $N$ of $\mathrm{H}_{\Phi}^{t}(M)$, the $R$-modules $\operatorname{Hom}_{R}\left(R / I, \mathrm{H}_{\Phi}^{t}(M) / N\right)$ and $\operatorname{Ext}_{R}^{1}\left(R / I, \mathrm{H}_{\Phi}^{t}(M) / N\right)$ are minimax. Let $N$ be a finitely generated $R$-module. We also prove that $\operatorname{Ext}_{R}^{j}\left(N, \mathrm{H}_{\Phi}^{i}(M)\right)$ and $\operatorname{Tor}_{j}^{R}\left(N, H_{\Phi}^{i}(M)\right)$ are $\Phi$-cominimax for all $i$ and $j$ whenever $M$ is minimax and $\mathrm{H}_{\Phi}^{i}(M)$ is $\mathrm{FD}_{\leq 1}$ (or weakly Laskerian) for all $i$.
\end{abstract}

Mathematics Subject Classification (2010). 13D45, 13E05, 14B15

Keywords. Local cohomology, $\mathrm{FD}_{\leq \mathrm{n}}$ modules, ETH-cominimax modules

\section{Introduction}

Throughout this paper $R$ is a commutative Noetherian ring with non-zero identity and $I$ is an ideal of $R$. For an $R$-module $M$, the $i^{t h}$ local cohomology module $M$ with respect to ideal $I$ is defined as

$$
\mathrm{H}_{I}^{i}(M) \cong \underset{n}{\lim } \operatorname{Ext}_{R}^{i}\left(R / I^{n}, M\right) .
$$

Grothendieck in [22] posed the following conjecture:

Conjecture 1.1. Let $M$ be a finitely generated $R$-module and $I$ an ideal of $R$. Then $\operatorname{Hom}_{R}\left(R / I, \mathrm{H}_{I}^{i}(M)\right)$ is finite for all $i \geq 0$.

This conjecture is not true in general as Hartshorne showed in [23], but some authors proved that for some number $t$, the module $\operatorname{Hom}_{R}\left(R / I, H_{I}^{t}(M)\right)$ is finite under some conditions. See [7, Theorem 3.3], [19, Theorem 6.3.9], [18, Theorem 2.1], [10, Theorem 2.6], [9, Theorem 2.3] and [4, Theorem 3.4]. Hartshorne also defined a module $M$ to be $I$-cofinite if $\operatorname{Supp}_{R}(M) \subseteq \mathrm{V}(I)$ and $\operatorname{Ext}_{R}^{i}(R / I, M)$ is finitely generated for all $i \geq 0$ and posed the following question:

Question 1.2. Let $M$ be a finite $R$-module and $I$ be an ideal of $R$. When are $\mathrm{H}_{I}^{i}(M)$ $I$-cofinite for all $i \geq 0$ ?

Email addresses: m-aghapour@araku.ac.ir

Received: 28.06.2017; Accepted: 12.02.2018 
This question was studied by several authors in [4,10,17,23, 25, 30-32].

There are some generalizations of the theory of ordinary local cohomology modules. The following is introduced by Bijan-Zadeh in [13].

Let $\Phi$ be a non-empty set of ideals of $R$. We call $\Phi$ a system of ideals of $R$ if, whenever $I_{1}, I_{2} \in \Phi$, then there is an ideal $J \in \Phi$ such that $J \subseteq I_{1} I_{2}$. For such a system, for every $R$-module $M$, one can define

$$
\Gamma_{\Phi}(M)=\{x \in M \mid I x=0 \text { for some } I \in \Phi\} .
$$

Then $\Gamma_{\Phi}(-)$ is a functor from $\mathscr{C}(R)$ to itself (where $\mathscr{C}(R)$ denotes the category of all $R$-modules and all $R$-homomorphisms). The functor $\Gamma_{\Phi}(-)$ is additive, covariant, $R$ linear and left exact. In [12], $\Gamma_{\Phi}(-)$ is denoted by $L_{\Phi}(-)$ and is called the "general local cohomology functor with respect to $\Phi$ ". For each $i \geq 0$, the $i$-th right derived functor of $\Gamma_{\Phi}(-)$ is denoted by $\mathrm{H}_{\Phi}^{i}(-)$. The functor $\mathrm{H}_{\Phi}^{i}(-)$ and $\underset{I \in \Phi}{\lim } \mathrm{H}_{I}^{i}(-)$ (from $\mathscr{C}(R)$ to itself) are naturally equivalent (see [13]). For an ideal $I$ of $R$, if $\Phi=\left\{I^{n} \mid n \in \mathbb{N}_{0}\right\}$, then the functor $\mathrm{H}_{\Phi}^{i}(-)$ coincides with the ordinary local cohomology functor $\mathrm{H}_{I}^{i}(-)$. It is shown that, the study of torsion theory over $R$ is equivalent to study the general local cohomology theory (see [12]).

As a special case of [35, Definition 2.1] and generalization of FSF modules (see [26, Definition 2.1]), in [4, Definition 2.1] the author of present paper and Bahmanpour introduced the class of $\mathrm{FD}_{\leq \mathrm{n}}$ modules. A module $M$ is said to be $\mathrm{FD}_{\leq \mathrm{n}}$ module, if there exists a finitely generated submodule $N$ of $M$ such that $\operatorname{dim} M / N \leq n$. For more details about properties of this class see [4, Lemma 2.3]. Note that the class of $\mathrm{FD}_{\leq-1}$ is the same as finitely generated $R$-modules. Recall that a module $M$ is a minimax module if there is a finitely generated submodule $N$ of $M$ such that the quotient module $M / N$ is Artinian. Minimax modules have been studied by Zöschinger in [37]. Note that for a complete Noetherian local ring, the class of minimax modules is the same as the class of Matlis reflexive modules (see [21] and [36]). Since the class of minimax modules is a generalization of Matlis reflexive modules, thus the study of minimax modules is as important as the study of Matlis reflexive modules.

Recall too that an $R$-module $M$ is called weakly Laskerian if $\operatorname{Ass}_{R}(M / N)$ is a finite set for each submodule $N$ of $M$. The class of weakly Laskerian modules introduced in [20]. In [5, Definition 3.1] the author of present paper and Bahmanpour introduced the concept of $\Phi$-cominimaxness of general local cohomology modules. The general local cohomology module $\mathrm{H}_{\Phi}^{j}(M)$ is defined to be $\Phi$-cominimax if there exists an ideal $I \in \Phi$ such that $\operatorname{Ext}_{R}^{i}\left(R / I, \mathrm{H}_{\Phi}^{j}(M)\right)$ is minimax, for all $i, j \geq 0$.

Recently many authors studied the minimaxness and cominimaxness of local cohomology modules and answered the Conjecture 1.1 and Question 1.2 in the class of minimax modules in some cases (see $[1,3,9,14,24,27,29]$ ). The purpose of this note is to make a suitable generalization of Conjecture 1.1 and Question 1.2 in terms of minimax modules instead of finitely generated modules for general local cohomology modules. In this direction in Section 2, we generalize [4, Theorem 3.4 and Corollaries 3.5 and 3.6] and [3, Theorem 2.7 and Corollaries 2.8 and 2.9]. More precisely, we shall show that:

Theorem 1.3. (See Theorem 2.10) Let $R$ be a Noetherian ring and $I \in \Phi$ an ideal of $R$. Let $t \in \mathbb{N}_{0}$ be an integer and $M$ an $R$-module such that $\operatorname{Ext}_{R}^{i}(R / I, M)$ are minimax for all $i \leq t+1$. Let the $R$-modules $\mathrm{H}_{\Phi}^{i}(M)$ are $\mathrm{FD}_{\leq 1}$ (or weakly Laskerian) $R$-modules for all $i<t$. Then, the following conditions hold:

(i) The R-modules $\mathrm{H}_{\Phi}^{i}(M)$ are $\Phi$-cominimax for all $i<t$.

(ii) For all $\mathrm{FD}_{\leq 0}$ (or minimax) submodule $N$ of $\mathrm{H}_{\Phi}^{t}(M)$, the $R$-modules

$$
\operatorname{Hom}_{R}\left(R / I, \mathrm{H}_{\Phi}^{t}(M) / N\right) \text { and } \operatorname{Ext}_{R}^{1}\left(R / I, \mathrm{H}_{\Phi}^{t}(M) / N\right)
$$

are minimax. 
Corollary 1.4. (See Corollary 2.11) Let $R$ be a Noetherian ring and $I \in \Phi$ an ideal of $R$. Let $M$ be an $R$-module such that $\operatorname{Ext}_{R}^{i}(R / I, M)$ are minimax for all $i$ and the $R$-modules $\mathrm{H}_{\Phi}^{i}(M)$ are $\mathrm{FD}_{\leq 1}$ (or weakly Laskerian) $R$-modules for all $i$. Then,

(i) the $R$-modules $\mathrm{H}_{\Phi}^{i}(M)$ are $\Phi$-cominimax for all $i$.

(ii) for any $i \geq 0$ and for any $\mathrm{FD}_{\leq 0}$ (or minimax) submodule $N$ of $\mathrm{H}_{\Phi}^{i}(M)$, the $R$ module $\mathrm{H}_{\Phi}^{i}(M) / N$ is $\Phi$-cominimax.

Hartshorne also asked the following question:

Question 1.5. Whether the category $\mathscr{M}(R, I)_{\text {cof }}$ of $I$-cofinite modules forms an Abelian subcategory of the category of all $R$-modules? That is, if $f: M \longrightarrow N$ is an $R$-module homomorphism of $I$-cofinite modules, are Ker $f$ and Coker $f$ I-cofinite?

With respect to this question, Hartshorne showed that if $I$ is a prime ideal of dimension one in a complete regular local ring $R$, then the answer to his question is positive. On the other hand, in [17], Delfino and Marley extended this result to arbitrary complete local rings. Recently, Kawasaki [28] generalized the Delfino and Marley's result for an arbitrary ideal $I$ of dimension one in a local ring $R$. Finally, Melkersson in [33] completely have removed local assumption on $R$. More recently, in [11] (resp. [4]) it is shown that Hartshorne's question is true for the category of all $I$-cofinite $R$-modules $M$ with $\operatorname{dim} M \leq 1$ (resp. the class of $I$ - cofinite $\mathrm{FD}_{\leq 1}$ modules), for all ideals $I$ in a commutative Noetherian ring $R$. Also in [27] (resp. [3]) it is proved that the same question is true for the category of all $I$-cominimax $R$-modules $M$ with $\operatorname{dim} M \leq 1$ (resp. the class of $I$ - cominimax $\mathrm{FD}_{\leq 1}$ modules), for all ideals $I$ in $R$. In this direction we introduced the concept of $I$-ETH-cominimax or ETH-cominimax modules with respect to $I$ in Definition 2.1. One of the main results of this section is to prove that the class of $I$-ETH-cominimax weakly Laskerian $\left(\mathscr{W} \mathscr{L}(R, I)_{\text {ethcom }}\right)$ and $I$-ETH-cominimax $\mathrm{FD}_{\leq 1}\left(\mathscr{F} \mathscr{D}^{1}(R, I)_{\text {ethcom }}\right)$ modules are Abelian category (see Theorem 2.13). Using this fact we generalize [24, Corollry 3.5] as below:

Corollary 1.6. (See Corollary 2.15) Let $\Phi$ be a system of ideals of a Noetherian ring $R, M$ a non-zero $I$-ETH-cominimax $R$-module such that $\mathrm{H}_{\Phi}^{i}(M)$ are $\mathrm{FD}_{\leq 1}$ (or weakly Laskerian) $R$-modules for all $i \geq 0$. Then for each finite $R$-module $N$, the $R$-modules $\operatorname{Ext}_{R}^{j}\left(N, \mathrm{H}_{\Phi}^{i}(M)\right)$ and $\operatorname{Tor}_{j}^{R}\left(N, \mathrm{H}_{\Phi}^{i}(M)\right)$ are $\Phi$-cominimax and $\mathrm{FD}_{\leq 1}$ (or weakly Laskerian) $R$-modules for all $i \geq 0$ and $j \geq 0$.

In Section 3 we prove that similar corollaries are true for local cohomology modules defined by a pair of ideals because it is a special case of local cohomology with respect to a system of ideals.

Throughout this paper, $R$ will always be a commutative Noetherian ring with non-zero identity and $I$ will be an ideal of $R$. We denote $\{\mathfrak{p} \in \operatorname{Spec} R: \mathfrak{p} \supseteq I\}$ by $V(I)$. For any unexplained notation and terminology we refer the reader to [15] and [16].

\section{ETH-cominimax modules with respect to an ideal}

The definitions of $E T H$-cofinite module and cominimax module with respect to an ideal ([2, Definitions 2.2] and [8, Definitions 3.1]), motivate the following definition.

Definition 2.1. An $R$-module $M$ is called $E T H$-cominimax with respect to an ideal $I$ of $R$ or $I$-ETH-cominimax if $\operatorname{Ext}_{R}^{i}(R / I, M)$ is a minimax $R$-module for all $i$.

Remark 2.2. Let $I$ be an ideal of $R$.

(i) All minimax $R$-modules, $E T H$-cofinite and cominimax $R$-modules with respect to ideal $I$ are $I$-ETH-cominimax.

(ii) Suppose $M$ is an $I$-torsion module, then $M$ is $I$-ETH-cominimax if and only if it is $I$-cominimax module. 
We claim that the class of $E T H$-cominimax modules with respect to an ideal is strictly larger than the class of $E T H$-cofinite modules and cominimax modules with respect to the same ideal. To do this, see the following examples.

Example 2.3. (i) Let $(R, \mathfrak{m})$ be a Noetherian local ring of dimension $d>0$. Let $M=$ $R \oplus E(R / \mathfrak{m})$. It is easy to see that $M$ is an $\mathfrak{m}$-ETH-cominimax $R$-module that is not $\mathfrak{m}$-cominimax.

(ii) Let $(R, \mathfrak{m})$ be a local ring and $\mathfrak{p}$ be a prime ideal of $R \operatorname{such}$ that $\operatorname{dim} R / \mathfrak{p}=1$, then it is easy to see that the $R$-module $\mathrm{E}(R / \mathfrak{p})$ is $\mathfrak{p}$-ETH-cominimax but it is not $\mathfrak{p}$-ETHcofinite.

The following lemma is also true and well-known for $I$-cofinite modules.

Lemma 2.4. If $0 \longrightarrow N \longrightarrow L \longrightarrow T \longrightarrow 0$ is exact and two of the modules in the sequence are I-ETH-cominimax, then so is the third one.

Lemma 2.5. Let $I$ be an ideal of a Noetherian ring $R$ and $M$ be an $R$-module such that $\operatorname{Supp}_{R}(M) \subseteq \operatorname{Max}(R)$. Then the following statements are equivalent:

(i) $M$ is I-ETH-cominimax.

(ii) The $R$-module $\operatorname{Hom}_{R}(R / I, M)$ is minimax.

Proof. (i) $\Longrightarrow$ (ii) follows by definition.

In order to prove (ii) $\Longrightarrow$ (i) note that

$$
\operatorname{Hom}_{R}\left(R / I, \Gamma_{I}(M)\right) \cong \operatorname{Hom}_{R}(R / I, M) .
$$

Since $\operatorname{Supp}_{R}\left(\Gamma_{I}(M)\right) \subseteq \operatorname{Max}(R)$, it is easy to see that $\operatorname{Hom}_{R}\left(R / I, \Gamma_{I}(M)\right)$ is an Artinian $R$-module and so by Melkersson's theorem [15, Theorem 7.1.2] $\Gamma_{I}(M)$ is also an Artinian $R$-module. On the other hand by [15, Theorem 6.1.2] $\mathrm{H}_{I}^{i}(M)=0$ for all $i \geq 1$. Thus the $R$-module $\mathrm{H}_{I}^{i}(M)$ is $I$-cominimax for all $i \geq 0$. Now by [32, Corollary 3.10], it follows that $\operatorname{Ext}_{R}^{i}(R / I, M)$ are minimax for all $i \geq 0$, as required.

Theorem 2.6. Let $I$ be an ideal of a Noetherian ring $R$ and $M$ be an $\mathrm{FD}_{\leq 0}$ (or minimax) $R$-module. Then the following statements are equivalent:

(i) $M$ is I-ETH-cominimax.

(ii) The $R$-module $\operatorname{Hom}_{R}(R / I, M)$ is minimax.

Proof. By definition there is a finitely generated submodule $N$ of $M$ such that $\operatorname{dim}(M / N) \leq$ 0 . Also, the exact sequence

$$
0 \rightarrow N \rightarrow M \rightarrow M / N \rightarrow 0,
$$

induces the following exact sequence

$$
\begin{aligned}
0 \longrightarrow \operatorname{Hom}_{R}(R / I, N) & \longrightarrow \operatorname{Hom}_{R}(R / I, M) \longrightarrow \operatorname{Hom}_{R}(R / I, M / N) \\
& \longrightarrow \operatorname{Ext}_{R}^{1}(R / I, N) .
\end{aligned}
$$

Whence, it follows that the $R$-modules $\operatorname{Hom}_{R}(R / I, M / N)$ is minimax. Therefore, in view of Lemma 2.5, the $R$-module $M / N$ is $I$-ETH-cominimax. Now it follows from the exact sequence $(*)$ and Lemma 2.4 that $M$ is $I-E T H$-cominimax.

We are now ready to state and prove the first main theorem of this section. The following theorem is a generalization of $[4,11$, Proposition 2.6] and [3,27, Proposition 2.4]. In fact, we remove $I$-torsion condition from these propositions. Note that $I$ is not dimension one too.

Lemma 2.7. Let $R$ be a Noetherian ring and $I$ be an ideal of $R$ (not necessary dimension one). Let $M$ be a non-zero $R$-module (not necessary $I$-torsion) such that $\operatorname{dim} M \leq 1$. Then the following conditions are equivalent: 
(i) $M$ is I-ETH-cominimax.

(ii) The $R$-modules $\operatorname{Hom}_{R}(R / I, M)$ and $\operatorname{Ext}_{R}^{1}(R / I, M)$ are minimax.

Proof. (ii) $\Longrightarrow$ (i) Using the exact sequence

$$
0 \rightarrow \Gamma_{I}(M) \rightarrow M \rightarrow M / \Gamma_{I}(M) \rightarrow 0
$$

we get the exact sequence

$$
\begin{gathered}
0 \longrightarrow \operatorname{Hom}_{R}\left(R / I, \Gamma_{I}(M)\right) \longrightarrow \operatorname{Hom}_{R}(R / I, M) \longrightarrow \operatorname{Hom}_{R}\left(R / I, M / \Gamma_{I}(M)\right) \longrightarrow \\
\operatorname{Ext}_{R}^{1}\left(R / I, \Gamma_{I}(M)\right) \longrightarrow \operatorname{Ext}_{R}^{1}(R / I, M) .
\end{gathered}
$$

Since $\operatorname{Hom}_{R}\left(R / I, M / \Gamma_{I}(M)\right)=0$, it follows that the $R$-modules

$$
\operatorname{Hom}_{R}\left(R / I, \Gamma_{I}(M)\right) \text { and } \operatorname{Ext}_{R}^{1}\left(R / I, \Gamma_{I}(M)\right)
$$

are minimax, and so in view of $\left[27\right.$, Proposition 2.4] the $R$-module $\Gamma_{I}(M)$ is $I$-cominimax. Now as the $R$-module $\operatorname{Ext}_{R}^{1}(R / I, M)$ is minimax, it follows from [6, Theorem 4.1 (c)] that the $R$-module $\operatorname{Hom}_{R}\left(R / I, \mathrm{H}_{I}^{1}(M)\right)$ is minimax. If $\mathfrak{p} \in \operatorname{Supp}_{R}\left(\mathrm{H}_{I}^{1}(M)\right) \subseteq \operatorname{Supp}_{R}(M)$, then

$$
\mathrm{H}_{I R_{\mathfrak{p}}}^{1}\left(M_{\mathfrak{p}}\right) \cong \mathrm{H}_{I}^{1}(M)_{\mathfrak{p}} \neq 0
$$

Since $\operatorname{dim} M \leq 1$, it is easy to see that $\operatorname{dim} R / \mathfrak{p}=0$ or $\operatorname{dim} R / \mathfrak{p}=1$. If $\operatorname{dim} R / \mathfrak{p}=1$ then $M_{\mathfrak{p}}$ is a zero dimensional $R_{\mathfrak{p}}$-module that implies $\mathrm{H}_{I R_{\mathfrak{p}}}^{1}\left(M_{\mathfrak{p}}\right)=0$ by using Grothendieck vanishing theorem [15, Theorem 6.1.2] which is a contradiction. Thus $\operatorname{dim} R / \mathfrak{p}=0$ and so $\mathfrak{p}$ is a maximal ideal. So we have the following inclusion

$$
\operatorname{Supp}_{R}\left(\operatorname{Hom}_{R}\left(R / I, \mathrm{H}_{I}^{1}(M)\right)\right) \subseteq \operatorname{Supp}_{R}\left(\mathrm{H}_{I}^{1}(M)\right) \subseteq \operatorname{Max} R .
$$

It is easy to see that the $R$-module $\operatorname{Hom}_{R}\left(R / I, \mathrm{H}_{I}^{1}(M)\right)$ is Artinian and so by [15, Theorem 7.1.2] the $R$-module $\mathrm{H}_{I}^{1}(M)$ is Artinian. Thus in view of Melkersson's theorem [15, Theorem 6.1.2] the $R$-module $\mathrm{H}_{I}^{i}(M)$ is $I$-cominimax for all $i \geq 0$. Now by [32, Corollary 3.10], it follows that $\operatorname{Ext}_{R}^{i}(R / I, M)$ are minimax for all $i \geq 0$, as required.

(i) $\Longrightarrow$ (ii) It is obviously true.

The following theorem is a generalization of [4, Theorem 3.1] and [3, Theorem 2.5] that in what follows the next theorem plays an important role.

Theorem 2.8. Let $R$ be a Noetherian ring and $I$ be an ideal of $R$. Let $M$ be an $\mathrm{FD}_{\leq 1} R$ module. Then $M$ is $I$-ETH-cominimax if and only if $\operatorname{Hom}_{R}(R / I, M)$ and $\operatorname{Ext}_{R}^{1}(R / I, M)$ are minimax.

Proof. By definition there is a finitely generated submodule $N$ of $M$ such that $\operatorname{dim}(M / N) \leq$ 1. Also, the exact sequence

$$
0 \rightarrow N \rightarrow M \rightarrow M / N \rightarrow 0
$$

induces the following exact sequence

$$
\begin{gathered}
0 \longrightarrow \operatorname{Hom}_{R}(R / I, N) \longrightarrow \operatorname{Hom}_{R}(R / I, M) \longrightarrow \operatorname{Hom}_{R}(R / I, M / N) \\
\longrightarrow \operatorname{Ext}_{R}^{1}(R / I, N) \longrightarrow \operatorname{Ext}_{R}^{1}(R / I, M) \longrightarrow \operatorname{Ext}_{R}^{1}(R / I, M / N) \longrightarrow \operatorname{Ext}_{R}^{2}(R / I, N) .
\end{gathered}
$$

Whence, it follows that the $R$-modules $\operatorname{Hom}_{R}(R / I, M / N)$ and $\operatorname{Ext}_{R}^{1}(R / I, M / N)$ are minimax. Therefore, in view of Proposition 2.7, the $R$-module $M / N$ is $I$-ETH-cominimax. Now it follows from the exact sequence $(*)$ that $M$ is $I$-ETH-cominimax.

The following lemma is needed in the proof of second main result of this paper.

Lemma 2.9. Let $I$ be an ideal of a Noetherian ring $R, M$ a non-zero $R$-module and $t \in \mathbb{N}_{0}$. Suppose that the $R$-module $\mathrm{H}_{\Phi}^{i}(M)$ is $I$-ETH-cominimax for all $i=0, \ldots, t-1$, and the $R$-modules $\operatorname{Ext}_{R}^{t}(R / I, M)$ and $\operatorname{Ext}_{R}^{t+1}(R / I, M)$ are minimax. Then the $R$-modules $\operatorname{Hom}_{R}\left(R / I, \mathrm{H}_{\Phi}^{t}(M)\right)$ and $\operatorname{Ext}_{R}^{1}\left(R / I, \mathrm{H}_{\Phi}^{t}(M)\right)$ are minimax. 
Proof. We use induction on $t$. The exact sequence

$$
0 \longrightarrow \Gamma_{\Phi}(M) \longrightarrow M \longrightarrow M / \Gamma_{\Phi}(M) \longrightarrow 0,
$$

induces the following exact sequence:

$$
\begin{gathered}
0 \longrightarrow \operatorname{Hom}_{R}\left(R / I, \Gamma_{\Phi}(M)\right) \longrightarrow \operatorname{Hom}_{R}(R / I, M) \longrightarrow \operatorname{Hom}_{R}\left(R / I, M / \Gamma_{\Phi}(M)\right) \\
\longrightarrow \operatorname{Ext}_{R}^{1}\left(R / I, \Gamma_{\Phi}(M)\right) \longrightarrow \operatorname{Ext}_{R}^{1}(R / I, M) .
\end{gathered}
$$

Since $\operatorname{Hom}_{R}\left(R / I, M / \Gamma_{\Phi}(M)\right)=0$ so

$$
\operatorname{Hom}_{R}\left(R / I, \Gamma_{\Phi}(M)\right) \text { and } \operatorname{Ext}_{R}^{1}\left(R / I, \Gamma_{\Phi}(M)\right)
$$

are minimax. Assume inductively that $t>0$ and that we have established the result for non-negative integers smaller than $t$. By applying the functor $\operatorname{Hom}_{R}(R / I,-)$ to the exact sequence (*), we can deduce that $\operatorname{Ext}_{R}^{j}\left(R / I, M / \Gamma_{\Phi}(M)\right)$ is minimax for $j=t, t+1$. On the other hand,

$$
\mathrm{H}_{I}^{0}\left(M / \Gamma_{\Phi}(M)\right)=0 \text { and } \mathrm{H}_{\Phi}^{j}\left(M / \Gamma_{\Phi}(M)\right) \cong \mathrm{H}_{\Phi}^{j}(M)
$$

for all $j>0$. Therefore we may assume that $\Gamma_{\Phi}(M)=0$. Let $E$ be an injective hull of $M$ and put $N=E / M$. Then $\operatorname{Hom}_{R}(R / I, E)=0=\Gamma_{\Phi}(E)$. Hence $\operatorname{Ext}_{R}^{j}(R / I, N) \cong \operatorname{Ext}_{R}^{j+1}(R / I, M)$ and $\mathrm{H}_{\Phi}^{j}(N) \cong \mathrm{H}_{\Phi}^{j+1}(M)$ for all $j \geq 0$. Now, the induction hypothesis yields that $\operatorname{Hom}_{R}\left(R / I, \mathrm{H}_{\Phi}^{t-1}(N)\right)$ and $\operatorname{Ext}_{R}^{1}\left(R / I, \mathrm{H}_{\Phi}^{t-1}(N)\right)$ are minimax and so $\operatorname{Hom}_{R}\left(R / I, \mathrm{H}_{\Phi}^{t}(M)\right)$ and $\operatorname{Ext}_{R}^{1}\left(R / I, \mathrm{H}_{\Phi}^{t}(M)\right)$ are minimax, as required.

We are now ready to state and prove the following main results (Theorem 2.10 and the Corollaries 2.11 and 2.12) which are extension of Bahmanpour-Naghipour's results in $[9,10]$ in terms of minimax modules, [14, Corollary 2.3], [1, Corollary 2.3], Hong Quy's result in [26], [4, Theorem 3.4 and Corollaries 3.5 and 3.6] and [3, Theorem 2.7 and Corollaries 2.8 and 2.9].

Theorem 2.10. Let $R$ be a Noetherian ring and $I \in \Phi$ an ideal of $R$. Let $t \in \mathbb{N}_{0}$ be an integer and $M$ an $R$-module such that $\operatorname{Ext}_{R}^{i}(R / I, M)$ are minimax for all $i \leq t+1$. Let the $R$-modules $\mathrm{H}_{\Phi}^{i}(M)$ are $\mathrm{FD}_{\leq 1}$ (or weakly Laskerian) $R$-modules for all $i<t$. Then, the following conditions hold:

(i) The R-modules $\mathrm{H}_{\Phi}^{i}(M)$ are $I$-ETH-cominimax (in particular $\Phi$-cominimax) for all $i<t$.

(ii) For all $\mathrm{FD}_{\leq 0}$ (or minimax) submodule $N$ of $\mathrm{H}_{\Phi}^{t}(M)$, the $R$-modules

$$
\operatorname{Hom}_{R}\left(R / I, \mathrm{H}_{\Phi}^{t}(M) / N\right) \text { and } \operatorname{Ext}_{R}^{1}\left(R / I, \mathrm{H}_{\Phi}^{t}(M) / N\right)
$$

are minimax. In particular the sets

$$
\operatorname{Ass}_{R}\left(\operatorname{Hom}_{R}\left(R / I, \mathrm{H}_{\Phi}^{t}(M) / N\right)\right) \text { and } \operatorname{Ass}_{R}\left(\operatorname{Ext}_{R}^{1}\left(R / I, \mathrm{H}_{\Phi}^{t}(M) / N\right)\right)
$$

are finite sets.

Proof. (i) We proceed by induction on $t$. In the case $t=0$ there is nothing to prove. So, let $t>0$ and the result has been proved for smaller values of $t$. By the inductive assumption, $\mathrm{H}_{\Phi}^{i}(M)$ is $I$-ETH-cominimax for $i=0,1, \ldots, t-2$. Hence by Lemma 2.9 and assumption, $\operatorname{Hom}_{R}\left(R / I, \mathrm{H}_{\Phi}^{t-1}(M)\right)$ and $\operatorname{Ext}_{R}^{1}\left(R / I, \mathrm{H}_{\Phi}^{t-1}(M)\right)$ are minimax. Therefore by Theorem 2.8, $\mathrm{H}_{\Phi}^{i}(M)$ is $I$-ETH-cominimax (in particular, $\Phi$-cominimax) for all $i<t$. This completes the inductive step.

(ii) In view of (i) and Lemma 2.9, $\operatorname{Hom}_{R}\left(R / I, \mathrm{H}_{\Phi}^{t}(M)\right)$ and $\operatorname{Ext}_{R}^{1}\left(R / I, \mathrm{H}_{\Phi}^{t}(M)\right)$ are minimax. On the other hand, according to Theorem 2.6, $N$ is $I$-ETH-cominimax. Now, the exact sequence 


$$
0 \longrightarrow N \longrightarrow \mathrm{H}_{\Phi}^{t}(M) \longrightarrow \mathrm{H}_{\Phi}^{t}(M) / N \longrightarrow 0
$$

induces the following exact sequence,

$$
\begin{gathered}
\operatorname{Hom}_{R}\left(R / I, \mathrm{H}_{\Phi}^{t}(M)\right) \longrightarrow \operatorname{Hom}_{R}\left(R / I, \mathrm{H}_{\Phi}^{t}(M) / N\right) \longrightarrow \operatorname{Ext}_{R}^{1}(R / I, N) \longrightarrow \\
\operatorname{Ext}_{R}^{1}\left(R / I, \mathrm{H}_{\Phi}^{t}(M)\right) \longrightarrow \operatorname{Ext}_{R}^{1}\left(R / I, \mathrm{H}_{\Phi}^{t}(M) / N\right) \longrightarrow \operatorname{Ext}_{R}^{2}(R / I, N) .
\end{gathered}
$$

Consequently

$$
\operatorname{Hom}_{R}\left(R / I, \mathrm{H}_{\Phi}^{t}(M) / N\right) \text { and } \operatorname{Ext}_{R}^{1}\left(R / I, \mathrm{H}_{\Phi}^{t}(M) / N\right)
$$

are minimax, as required.

The following corollaries answer to Hartshorne's question in terms of cominimax modules.

Corollary 2.11. Let $R$ be a Noetherian ring and $I \in \Phi$ an ideal of $R$. Let $M$ be an $I$ ETH-cominimax R-module such that the $R$-modules $\mathrm{H}_{\Phi}^{i}(M)$ are $\mathrm{FD}_{\leq 1}$ (or weakly Laskerian) $R$-modules for all $i$. Then,

(i) the R-modules $\mathrm{H}_{\Phi}^{i}(M)$ are I-ETH-cominimax (in particular, $\Phi$-cominimax) for all $i$.

(ii) for any $i \geq 0$ and for any $\mathrm{FD}_{\leq 0}$ (or minimax) submodule $N$ of $\mathrm{H}_{\Phi}^{i}(M)$, the $R$ module $\mathrm{H}_{\Phi}^{i}(M) / N$ is I-ETH-cominimax (in particular, $\Phi$-cominimax).

Proof. (i) Clear.

(ii) In view of (i) the $R$-module $\mathrm{H}_{\Phi}^{i}(M)$ is $I$-ETH-cominimax for all $i$. Hence the $R$ module $\operatorname{Hom}_{R}(R / I, N)$ is minimax, and so it follows from Lemma 2.6 that $N$ is $I$-ETHcominimax. Now, the exact sequence

$$
0 \longrightarrow N \longrightarrow \mathrm{H}_{\Phi}^{i}(M) \longrightarrow \mathrm{H}_{\Phi}^{i}(M) / N \longrightarrow 0
$$

and Lemma 2.4 implies that the $R$-module $\mathrm{H}_{\Phi}^{i}(M) / N$ is $I$-ETH-cominimax.

The following corollary is a generalization of [10, Corollary 2.7].

Corollary 2.12. Let $R$ be a Noetherian ring and $I \in \Phi$ an ideal of $R$. Let $M$ be an $I$-ETH-cominimax $R$-module such that $\operatorname{dim} R / I \leq 1$ for all $I \in \Phi$. Then,

(i) the R-modules $\mathrm{H}_{\Phi}^{i}(M)$ are I-ETH-cominimax (in particular, $\Phi$-cominimax) for all $i$.

(ii) for any $i \geq 0$ and for any $\mathrm{FD}_{\leq 0}$ (or minimax) submodule $N$ of $\mathrm{H}_{\Phi}^{i}(M)$, the $R$ module $\mathrm{H}_{\Phi}^{i}(M) / N$ is I-ETH-cominimax (in particular, $\Phi$-cominimax).

Proof. (i) Since by [13, Lemma 2.1],

$$
\mathrm{H}_{\Phi}^{i}(M) \cong \underset{I \in \Phi}{\lim _{I}} \mathrm{H}_{I}^{i}(M),
$$

it is easy to see that $\operatorname{Supp}_{R}\left(\mathrm{H}_{\Phi}^{i}(M)\right) \subseteq \bigcup_{I \in \Phi} \operatorname{Supp}_{R}\left(\mathrm{H}_{I}^{i}(M)\right)$ and therefore

$$
\operatorname{dimSupp} \mathrm{H}_{\Phi}^{i}(M) \leq \sup \left\{\operatorname{dimSupp} \mathrm{H}_{I}^{i}(M) \mid I \in \Phi\right\} \leq 1,
$$

thus $\mathrm{H}_{\Phi}^{i}(M)$ is $\mathrm{FD}_{\leq 1} R$-module and the assertion follows by Corollary 2.11 (i).

(ii) Proof is the same as 2.11 (ii).

One of the main results of this section is to prove that for an arbitrary ideal $I$ of a Noetherian ring $R$, the category of $\mathscr{W} \mathscr{L}(R, I)_{\text {ethcom }}$ and $\mathscr{F} \mathscr{D}^{1}(R, I)_{\text {ethcom }}$ modules are Abelian category. 
Theorem 2.13. Let $I$ be an ideal of a Noetherian ring $R$. Let $\mathscr{W} \mathscr{L}(R, I)_{\text {ethcom }}$ (resp. $\left.\mathscr{F} \mathscr{D}^{1}(R, I)_{\text {ethcom}}\right)$ denote the category of I-ETH-cominimax weakly Laskerian (resp. the category of $I$-ETH-cominimax $\left.\mathrm{FD}_{\leq 1}\right) R$-modules. Then $\mathscr{W} \mathscr{L}(R, I)_{\text {ethcom }}$ (resp. $\left.\mathscr{F} \mathscr{D}^{1}(R, I)_{\text {ethcom }}\right)$ is an Abelian category.

Proof. We prove theorem for the $\mathscr{W} \mathscr{L}(R, I)_{\text {ethcom }}$ case and by using the same proof, the $\mathscr{F} \mathscr{D}^{1}(R, I)_{\text {ethcom }}$ case follows.

Let $M, N \in \mathscr{W} \mathscr{L}(R, I)_{\text {ethcom }}$ and let $f: M \longrightarrow N$ be an $R$-homomorphism. It is enough to show that the $R$-modules Ker $f$ and Coker $f$ are $I$-ETH-cominimax.

To this end, the exact sequence

$$
0 \longrightarrow \operatorname{Ker} f \longrightarrow M \longrightarrow \operatorname{Im} f \longrightarrow 0,
$$

induces an exact sequence

$$
\begin{gathered}
0 \longrightarrow \operatorname{Hom}_{R}(R / I, \operatorname{Ker} f) \longrightarrow \operatorname{Hom}_{R}(R / I, M) \longrightarrow \operatorname{Hom}_{R}(R / I, \operatorname{Im} f) \\
\longrightarrow \operatorname{Ext}_{R}^{1}(R / I, \operatorname{Ker} f) \longrightarrow \operatorname{Ext}_{R}^{1}(R / I, M),
\end{gathered}
$$

that implies the $R$-modules $\operatorname{Hom}_{R}(R / I, \operatorname{Ker} f)$ and $\operatorname{Ext}_{R}^{1}(R / I, \operatorname{Ker} f)$ are minimax. Since Ker $f$ is a weakly Laskerian and so $\mathrm{FD}_{\leq 1} R$-module, therefore it follows from Theorem 2.8 that Ker $f$ is $I$-ETH-cominimax. Now, the assertion follows from the following exact sequences

$$
0 \longrightarrow \operatorname{Ker} f \longrightarrow M \longrightarrow \operatorname{Im} f \longrightarrow 0
$$

and

$$
0 \longrightarrow \operatorname{Im} f \longrightarrow N \longrightarrow \text { Coker } f \longrightarrow 0
$$

The following corollaries are generalization of [4, Corolaries 3.8 and 3.9] and [24, Theorem 3.4].

Corollary 2.14. Let $R$ be a Noetherian ring and $I$ a proper ideal of $R$. Let $M$ is a non-zero $I$-ETH-cominimax and weakly Laskerian (resp. I-ETH-cominimax $\mathrm{FD}_{\leq 1} \quad R$-module) $R$ module. Then, the $R$-modules $\operatorname{Ext}_{R}^{i}(N, M)$ and $\operatorname{Tor}_{i}^{R}(N, M)$ are I-ETH-cominimax and weakly Laskerian (resp. I-ETH-cominimax $\mathrm{FD}_{\leq 1}$ ) $R$-modules, for all finitely generated $R$-modules $N$ and all integers $i \geq 0$.

Proof. Since $N$ is finitely generated it follows that $N$ has a free resolution of finitely generated free modules. Now the assertion follows using Theorem 2.13 and computing the modules $\operatorname{Tor}_{i}^{R}(N, M)$ and $\operatorname{Ext}_{R}^{i}(N, M)$, by this free resolution.

Corollary 2.15. Let $\Phi$ be a system of ideals of a Noetherian ring $R, M$ a non-zero IETH-cominimax $R$-module such that $\mathrm{H}_{\Phi}^{i}(M)$ are $\mathrm{FD}_{\leq 1}$ (or weakly Laskerian) $R$-modules for all $i \geq 0$. Then for each finite $R$-module $N$, the $R$-modules $\operatorname{Ext}_{R}^{j}\left(N, \mathrm{H}_{\Phi}^{i}(M)\right)$ and $\operatorname{Tor}_{j}^{R}\left(N, \mathrm{H}_{\Phi}^{i}(M)\right)$ are $\Phi$-cominimax and $\mathrm{FD}_{\leq 1}$ (or weakly Laskerian) $R$-modules for all $i \geq 0$ and $j \geq 0$.

Proof. Apply Corollaries 2.14 and 2.11 .

\section{Cominimaxness of local cohomology defined by a pair of ideals}

As a special case of general local cohomology and generalization of ordinary local cohomology modules, R. Takahashi, Y. Yoshino, and T. Yoshizawa [34], introduced local cohomology modules with respect to a pair of ideals. The $(I, J)$-torsion submodule $\Gamma_{I, J}(M)$ of $M$ is a submodule of $M$ consists of all elements $x$ of $M$ with $\operatorname{Supp}(R x) \subseteq W(I, J)$, in which

$$
W(I, J)=\left\{\mathfrak{p} \in \operatorname{Spec}(R) \mid I^{n} \subseteq \mathfrak{p}+J \text { for an integer } n \geq 1\right\} .
$$


For an integer $i$, the $i$-th local cohomology functor $\mathrm{H}_{I, J}^{i}$ with respect to $(I, J)$ is the $i$-th right derived functor of $\Gamma_{I, J}$. The $R$-module $\mathrm{H}_{I, J}^{i}(M)$ is called the $i$-th local cohomology module of $M$ with respect to $(I, J)$. In the case $J=0, \mathrm{H}_{I, J}^{i}(-)$ coincides with the ordinary local cohomology functor $\mathrm{H}_{I}^{i}(-)$. Also, we are concerned with the following set of ideals of $R$ :

$$
\tilde{W}(I, J)=\left\{\mathfrak{a} \unlhd R \mid I^{n} \subseteq \mathfrak{a}+J \text { for an integer } n \geq 0\right\} .
$$

The definition of cominimax module with respect to an ideal ([8, Definition 2.1]) motivates the following definition.

Definition 3.1. An $R$-module $M$ is called $(I, J)$-cominimax if $\operatorname{Supp}_{R}(M) \subseteq W(I, J)$ and $\operatorname{Ext}_{R}^{i}(R / I, M)$ is a minimax $R$-module, for all $i \geq 0$.

Remark 3.2. Let $I$ and $J$ be two ideals of $R$. Replacing $\Phi$ by $\tilde{W}(I, J), \mathrm{H}_{\Phi}^{i}(M)$ by $\mathrm{H}_{I, J}^{i}(M)$ and $\Phi$-cominimax module by $(I, J)$-cominimax module, the Theorem 2.10 and Corollaries 2.11, 2.12 and 2.15 are true for local cohomology modules defined by a pair of ideals. Because by [34, Definition 3.1 and Theorem 3.2], it is easy to see that the local cohomology modules defined by a pair of ideals is a special case of local cohomology modules with respect to a system of ideals.

Acknowledgment. The author likes to thank the referee for his/her careful reading and many helpful suggestions on this paper.

\section{References}

[1] A. Abbasi, D. Hasanzadeh-Lelekami and H. Roshan-Shokalgourabi, Some results on the local cohomology of minimax modules, Czechoslovak Math. J. 64 (2), 327-333, 2014.

[2] M. Aghapournahr, On cofiniteness of local cohomology modules for a pair of ideals for small dimensions, J. Algebra Appl. 17 (2), 1850020, 2018.

[3] M. Aghapournahr, Cominimaxness of local cohomology modules, Czechoslovak Math. J. 69 (1), 75-86, 2019.

[4] M. Aghapournahr and K. Bahmanpour, Cofiniteness of weakly Laskerian local cohomology modules, Bull. Math. Soc. Sci. Math. Roumanie 105 (4), 347-356, 2014.

[5] M. Aghapournahr and K. Bahmanpour, Cofiniteness of general local cohomology modules for small dimensions, Bull. Korean Math. Soc. 53 (5), 1341-1352, 2016.

[6] M. Aghapournahr, L. Melkersson, A natural map in local cohomology, Ark. Mat. 48 (2), 243-251, 2010.

[7] J. Asadollahi, K. Khashyarmanesh and Sh. Salarian, A generalization of the cofiniteness problem in local cohomology modules, J. Aust. Math. Soc. 75 (3), 313-324, 2003.

[8] J. Azami, R. Naghipour, and B. Vakili, Finiteness properties of local cohomology modules for a-minimax modules, Proc. Amer. Math. Soc. 137 (2), 439-448, 2009.

[9] K. Bahmanpour and R. Naghipour, On the cofiniteness of local cohomology modules, Proc. Amer. Math. Soc. 136 (2008), 2359-2363.

[10] K. Bahmanpour and R. Naghipour, Cofiniteness of local cohomology modules for ideals of small dimension, J. Algebra 321 (7), 1997-2011, 2009.

[11] K. Bahmanpour, R. Naghipour and M. Sedghi, On the category of cofinite modules which is Abelian, Proc. Amer. Math. Soc. 142 (4), 1101-1107, 2014.

[12] M.H. Bijan-Zadeh, Torsion theory and local cohomology over commutative Noetherian ring, J. London Math. Soc. 19 (3), 402-410, 1979.

[13] M.H. Bijan-Zadeh, A common generalization of local cohomology theories, Glasgow Math. J. 21 (1), 173-181, 1980.

[14] K. Borna Lorestani, P. Sahandi and S. Yassemi, Artinian local cohomology modules, Canad. Math. Bull. 50 (4), 598-602, 2007. 
[15] M.P. Brodmann and R.Y. Sharp Local cohomology-An algebraic introduction with geometric applications, Cambridge. Univ. Press, 1998.

[16] W. Bruns and J. Herzog, Cohen Macaulay Rings, in: Cambridge Studies in Advanced Mathematics, 39, Cambridge Univ. Press, Cambridge, UK, 1993.

[17] D. Delfino and T. Marley, Cofinite modules and local cohomology, J. Pure Appl. Algebra 121 (1), 45-52, 1997.

[18] M.T. Dibaei and S. Yassemi, Associated primes and cofiniteness of local cohomology modules, Manuscripta Math. 117 (2), 199-205, 2005.

[19] M.T. Dibaei and S. Yassemi, Associated primes of the local cohomology modules, in: Abelian groups, rings, modules and homological algebra, 49-56, Chapman and Hall/CRC, 2006.

[20] K. Divaani-Aazar and A. Mafi, Associated primes of local cohomology modules, Proc. Amer. Math. Soc. 133 (3), 655-660, 2005.

[21] E. Enochs, Flat covers and flat cotorsion modules, Proc. Amer. Math. Soc. 92 (2), 179-184, 1984.

[22] A. Grothendieck, Cohomologie locale des faisceaux cohérents et théorèmes de Lefschetz locaux et globaux (SGA2), North-Holland, Amsterdam, 1968.

[23] R. Hartshorne, Affine duality and cofiniteness, Invent. Math. 9 (2), 145-164, 1970.

[24] D. Hasanzadeh-Lelekami, H. Roshan-Shokalgourabi, Extension functors of cominimax modules, Comm. Algebra 45 (2), 621-629, 2017.

[25] C. Huneke and J. Koh, Cofiniteness and vanishing of local cohomology modules, Math. Proc. Cambridge Philos. Soc. 110 (3), 421-429, 1991.

[26] P. Hung Quy, On the finiteness of associated primes of local cohomology modules, Proc. Amer. Math. Soc. 138 (6), 1965-1968, 2010.

[27] Y. Irani, Cominimaxness with respect to ideals of dimension one, Bull. Korean Math. Soc. 54 (1), 289-298, 2017.

[28] K.I. Kawasaki, On a category of cofinite modules which is Abelian, Math. Z. 269 (1-2), 587-608, 2011.

[29] A. Mafi, On the local cohomology of minimax modules, Bull. Korean Math. Soc. 48 (6), 1125-1128, 2011.

[30] T. Marley and J.C. Vassilev, Cofiniteness and associated primes of local cohomology modules, J. Algebra 256 (1), 180-193, 2002.

[31] L. Melkersson, Properties of cofinite modules and applications to local cohomology, Math. Proc. Cambridge Philos. Soc. 125 (3), 417-423, 1999.

[32] L. Melkersson, Modules cofinite with respect to an ideal, J. Algebra 285 (2), 649-668, 2005.

[33] L. Melkersson, Cofiniteness with respect to ideals of dimension one, J. Algebra $\mathbf{3 7 2}$, 459-462, 2012.

[34] R. Takahashi, Y. Yoshino and T. Yoshizawa, Local cohomology based on a nonclosed support defined by a pair of ideals, J. Pure Appl. Algebra 213 (4), 582-600, 2009.

[35] T. Yoshizawa, Subcategories of extension modules by subcategories, Proc. Amer. Math. Soc. 140 (7), 2293-2305, 2012.

[36] T. Zink, Endlichkeitsbedingungen für Moduln über einem Noetherschen Ring, Math. Nachr. 64 (1), 239-252, 1974.

[37] H. Zöschinger, Minimax Moduln, J. Algebra 102 (1), 1-32, 1986. 